\title{
Controller Tuning by Bayesian Optimization An Application to a Heat Pump
}

\section{Conference Paper}

\section{Author(s):}

Khosravi, Mohammad; Eichler, Annika (D); Schmid, Nicolas (D); Heer, Philipp; Smith, Roy (D)

Publication date:

2019

\section{Permanent link:}

https://doi.org/10.3929/ethz-b-000373882

\section{Rights / license:}

In Copyright - Non-Commercial Use Permitted

Originally published in:

https://doi.org/10.23919/ECC.2019.8795801 


\title{
Controller Tuning by Bayesian Optimization An Application to a Heat Pump
}

\author{
Mohammad Khosravi ${ }^{1}$, Annika Eichler ${ }^{1}$, Nicolas Schmid ${ }^{1}$, Philipp Heer ${ }^{2}$, Roy S. Smith ${ }^{1}$
}

\begin{abstract}
In this paper, we consider the problem of controller tuning for an operating unit in a building energy system. As an illustrative plant example we focus on a heat pump. Since the plant is in use, the tuning method is supposed to not intervene with its operation. Moreover, the tuning procedure is supposed to be online, model-free, based only on historical data and needs to provide safety guarantees of the plant in operation. In this regard, we formulate the problem as a black-box optimization and adopt safe Bayesian optimization approaches for controller parameter tuning. These approaches are relatively new to the control community and not intensively studied in control applications. Meanwhile, the underlying systems are often expensive and performing relevant experiments is time consuming. Therefore, a crucial step prior to implementation in reality is validating the methods in simulation to verify their applicability. Toward this end, we derive a physical-based model for the heat pump and identify the unknown parameters using gray-box identification methods. Given the simulation model, we tune the controller parameters in simulation for optimal performance while considering safety constraints of the system.
\end{abstract}

\section{INTRODUCTION}

Nearly $40 \%$ of the total energy is consumed by the commercial and residential building sector [6]. Optimizing energy systems in buildings can yield in a significant reduction in the corresponding consumption and the total energy demand. The control system implemented in buildings is structured and designed hierarchically with a central supervision control layer and several lower-level control layers below [4]. In the higher layers advanced automation strategies such as model predictive controls are designed [11] while in the lower layers less complicated control schemes such as P, PI and PID controllers are implemented. For operational performance all levels must be finely tuned. Here, we will focus on the lower level with the corresponding PID controllers. One can restate the problem of tuning the controller parameters as optimizing a properly designed metric which induces the system performance in its closedloop operation. In many industrial plants, tuning of the controllers is done based on manual and time consuming approaches like trial-and-error procedures and rules of thumb methods [1]. These methods are simple but do not providing optimal performance. Additionally, these approaches require specific experiments or modification of the plant, which may not be possible in many situations. Moreover, most of

\footnotetext{
*This research project is part of the Swiss Competence Center for Energy Research SCCER FEEB\&D of the Swiss Innovation Agency Innosuisse.

${ }^{1}$ The authors are with Automatic Control Lab, ETH Zurich, Switzerland \{khosravm, echlean, rsmith\}econtrol.ee.ethz.ch, nicolas_schmidemsn. com

2 The author is with EMPA, Dübendorf, Zurich, Switzerland philipp.heer@empa.ch
}

these methods are designed for linear time invariant systems and specific performance metrics [5], [3]. Accordingly, we require an automated tuning method, which is operating stably and safely in closed-loop, is capable of considering general performance metrics, and is based on historical data.

This problem is formulated as a Bayesian optimization problem [10] with safety constraints [12], [2]. In these methods, an exploration-exploitation trade-off is employed to learn the performance as a function of the controller parameters and meanwhile find the optimizer, both in a safe manner. An alternative approach is active learning, where the performance function is learned by safely and vastly sampling from the space of controller parameters [9], [8] and then, the optimizer and the optimal value are derived. Bayesian optimization formulations have been successfully utilized for many problems such as inverted pendulums, multi-armed bandit problems, robotics, combustion engines, etc. [9], [12], [8], [2]. However, they are not studied well for controller tuning in building energy systems and other thermodynamical cases. These types of systems are relatively slow and extensive experimentation is prohibitively time consuming. This highlights the importance of testing tuning methods for these systems in simulation. This way we can prestudy the performance of these methods for controller tuning for these type of plants. Moreover, we can compare different aspects of these approaches such as choices of algorithms, performance metrics, safety indicators, convergence time, Gaussian process kernels and hyper-parameters.

In this paper, we consider a heat pump in the NEST building, Empa, Zurich, Switzerland with the focus on the condenser part. First, we model the plant as a gray-box using laws of physics and thermodynamics. Using historical data, by solving a prediction error minimization problem, we estimate the parameters and derive the dynamics of the plant. This provides us a model representing the approximate behavior of the real plant. We use two Bayesian optimization approaches, safe active learning [9] and safe Bayesian optimization [2], for tuning the controller parameters of the system in simulation using the identified model. A performance metric for the desired behavior of the closedloop system and a constraint to guarantee safety in the tuning process are introduced.

\section{ModELING AND IDENTIFICATION A. Structure of Plant and Operational Modes}

General Scheme: The plant is a heat pump connected to two external flow circuits, called the secondary circuits. One of the external circuits corresponds to the heating side of 


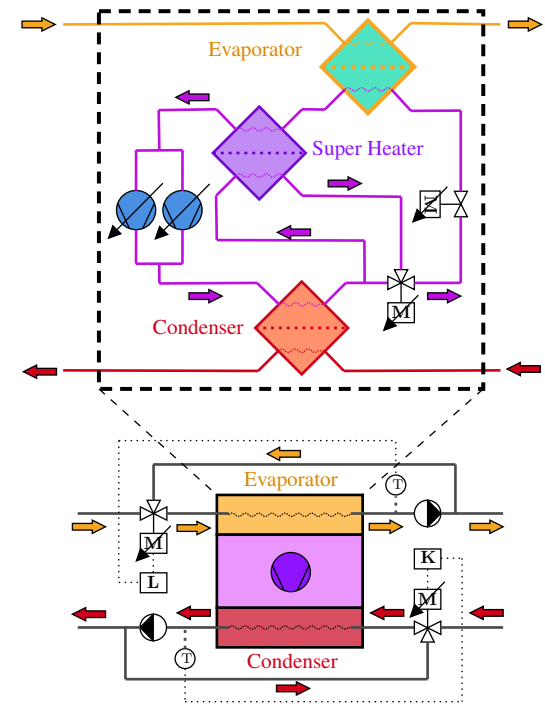

Fig. 1. The schematics of a heat pump with internal and external circuits. At the top, the heat pump scheme is magnified. As it is shown, in the internal loop of the heat pump, there are two compressors (shown in blue), a controlled expansion valve, a valve controlling the flow rate of refrigerant and three internal heat exchangers, called as evaporator (orange), super heater (purple) and condenser (red).

the heat pump, where water is circulating (shown in red in Figure 1), and the other one corresponds to the cooling side, where glycol is circulating (shown in orange in Figure 1).

Primary circuit: The heat pump itself has an internal flow circuit, called primary circuit where a refrigerant is circulating. As shown in Figure 1, the internal circuit consists of two compressors, a controlled expansion valve, a valve controlling the flow rate of refrigerant and three internal heat exchangers, labeled as evaporator, condenser and superheater. The evaporator transfers the heat content of the glycol to the refrigerant. Similarly, the condenser transfers the heat content of the refrigerant to the water. The super-heater improves the heating cycle by using the remaining heat content of refrigerant after it passes through the condenser and also controlling the heat transfer process via the internal compressors and valves.

Secondary circuits: At the condenser side, there exists a return flow pipe, which returns the heated water to mix it with the incoming water before entering the heat pump. This mixing is controlled via a mixing valve. As shown in Figure 1 , in order to guarantee the return flow, a pump is placed after the outlet. This pump maintains a pressure to provide a flow to the mixing valve. The temperature set point of the mixing valve is controlled via a PI-controller. The controller should be tuned so that the output temperature at the condenser tracks a given temperature reference. At the cooling side of the heat pump, a similar structure regulates the output temperature at the evaporator.

Heat pump operation: The heat pump is driven in cyclic operation, switching between operation mode and nonoperation mode. The decision on switching between these modes is done by a higher level supervisory controller and depends on the demand for heated water or cooled glycol.
Once, the system is turned on, the controlled mixing valves are used in order to control the output temperature of each side to a constant reference signal, which is the same in each operation cycle. We define as an operation cycle the time from switching the heat pump on until the next shut down and model the plant only within this operation mode.

\section{B. Modeling of the Plant}

For modeling we mainly focus on the heating side of the heat pump. Let $T_{i}$ and $T_{o}$ denote the temperature of water before and after passing through the condenser, respectively. Also, let $\mathrm{T}$ be the average temperature of water inside the condenser and $T_{c}$ the average temperature of the refrigerant in the condenser. Based on the second law of thermodynamics, one can write the first order dynamics as

$$
\frac{\mathrm{d}}{\mathrm{d} t}\left(\rho c_{p} V \mathrm{~T}\right)=\rho c_{p}\left(\mathrm{~T}_{\mathrm{i}}-\mathrm{T}_{\mathrm{o}}\right) \mathrm{F}_{\mathrm{i}}+Q,
$$

where $Q$ is the heat power transfered from the refrigerant side of the condenser, $\mathrm{F}_{\mathrm{i}}$ is the flow rate of water, $V$ is the internal volume of the condenser, $\rho$ is the density of water and $c_{p}$ is the specific heat of water. The heat power transfered, $Q$, is proportional to $T_{c}-T$. Additionally, we assume that $T$ is a convex combination of $\mathrm{T}_{\mathrm{i}}$ and $\mathrm{T}_{\mathrm{o}}$, i.e., for some constant $\theta_{1}$ in $[0,1]$, one has $\mathrm{T}=\left(1-\theta_{1}\right) \mathrm{T}_{\mathrm{i}}+\theta_{1} \mathrm{~T}_{\mathrm{o}}$. Accordingly, one can write $T_{0}$ in terms of $T$ and $T_{i}$ as

$$
\mathrm{T}_{\mathrm{o}}=\frac{1}{\theta_{1}}\left(\mathrm{~T}-\left(1-\theta_{1}\right) \mathrm{T}_{\mathrm{i}}\right) \text {. }
$$

Assuming that the volume, the density of water and its specific heat coefficients are constant, we can obtain the dynamics of $\mathrm{T}$ from (1) as

$$
\frac{\mathrm{d}}{\mathrm{d} t} \mathrm{~T}=\frac{\rho c_{p}}{\theta_{1}}\left(\mathrm{~T}_{\mathrm{i}}-\mathrm{T}\right) \mathrm{F}_{\mathrm{i}}+\frac{1}{\rho c_{p} V} Q .
$$

Similar to (3), one can derive first order dynamics for $T_{c}$. However, since the internal circuit as well as the secondary circuit, corresponding to the glycol flow, are both operating in closed loop, we assume that $\mathrm{T}_{\mathrm{c}}$ is controlled and has autonomous dynamics with an additive disturbance term according to the heat power, $Q$, transferred . More precisely, we model the dynamics of $\mathrm{T}_{\mathrm{c}}$ as

$$
\frac{\mathrm{d}}{\mathrm{d} t} \mathrm{~T}_{\mathrm{c}}=\Phi\left(\mathrm{T}_{\mathrm{c}}\right)-\gamma Q
$$

where $\gamma$ is a positive real scalar. Here, for the case of simplicity, we neglect heat losses and also assume that $\Phi$ is an affine function with respect to $\mathrm{T}_{\mathrm{c}}$. Setting a sampling time $T_{s}$, one can discretize the dynamics given in (3) and (4) using forward Euler method. Since $Q$ is proportional to $\mathrm{T}-\mathrm{T}_{\mathrm{c}}$, the resulting discrete time dynamics are

$$
\begin{aligned}
& \mathrm{T}^{+}=\left(1-\frac{\theta_{2}}{\theta_{1}} \mathrm{~F}_{\mathrm{i}}-\theta_{3}\right) \mathrm{T}+\frac{\theta_{2}}{\theta_{1}} \mathrm{~F}_{\mathrm{i}} \mathrm{T}_{\mathrm{i}}+\theta_{3} \mathrm{~T}_{\mathrm{c}}, \\
& \mathrm{T}_{\mathrm{c}}^{+}=\theta_{4}+\theta_{5} \mathrm{~T}_{\mathrm{c}}+\theta_{6} \mathrm{~T},
\end{aligned}
$$

where $\theta_{2}, \theta_{3}, \theta_{4}, \theta_{5}$ and $\theta_{6}$ are positive scalars. Note that these parameters depend on $T_{s}$.

Denote the flow rate of water passing through the heat pump and the flow rate of water in the returning pipe by 
$F_{i}$ and $F_{r}$, respectively. Also, let $F_{d}$ denote the flow rate of water entering the plant. Conservation of mass implies $F_{d}=$ $F_{i}-F_{r}$. Assuming a delay of one sampling time between the water temperature at the valve and at the inlet of the condenser, one can obtain the dynamics for temperature $T_{i}$ as a result of the mixing of fluids in the valve as

$$
\mathrm{T}_{\mathrm{i}}^{+}=\frac{\mathrm{F}_{\mathrm{d}}}{\mathrm{F}_{\mathrm{d}}+\mathrm{F}_{\mathrm{r}}} \mathrm{T}_{\mathrm{d}}+\frac{\mathrm{F}_{\mathrm{r}}}{\mathrm{F}_{\mathrm{d}}+\mathrm{F}_{\mathrm{r}}} \mathrm{T}_{\mathrm{r}},
$$

where $T_{r}$ is the temperature of returning water before the mixing valve and $T_{d}$ is the temperature of the water entering the plant. Due to the inertia of water, $\mathrm{T}_{\mathrm{r}}$ is a delayed version of $\mathrm{T}_{\mathrm{o}}$. More precisely, for any time instant $k$, one has

$$
\mathrm{T}_{\mathrm{r}}(k)=\mathrm{T}_{\mathrm{o}}(k-d),
$$

where $d$ is a constant positive integer representing the number of samples corresponding to the delay. Here, for the case of simplicity we neglect the heat loss in the pipe and also assume that the delay is independent of time and other introduced variables.

Define $u$ as the set point of the valve, which takes values in $[0,1]$ and determines how much the valve is open. When the valve is completely closed, i.e., $u=0$, the return flow is zero. If the valve is completely open, i.e., $u=1$, the return flow significantly affects and suppresses the flow $F_{d}$. This depends on the operation of the pump and the resulting pressure difference. Therefore, the flows $F_{r}$ and $F_{d}$ depend on the set point of the valve $u$. More precisely, it is assumed that there exist functions $\mathrm{f}_{\mathrm{d}}, \mathrm{f}_{\mathrm{r}}:[0,1] \rightarrow \mathbb{R}$ such that, at any time instant $k$, we have that

$$
\begin{aligned}
& \mathrm{F}_{\mathrm{d}}(k)=\mathrm{f}_{\mathrm{d}}(u(k))+\delta \mathrm{F}_{\mathrm{d}}(k), \\
& \mathrm{F}_{\mathrm{r}}(k)=\mathrm{f}_{\mathrm{r}}(u(k))+\delta \mathrm{F}_{\mathrm{r}}(k),
\end{aligned}
$$

where $\delta \mathrm{F}_{\mathrm{d}}(k)$ and $\delta \mathrm{F}_{\mathrm{r}}(k)$ denote the disturbances. These disturbances will be of major importance for the controller tuning from Section III on, since they are the reason for the noise we have in the simulations.

Let $k$ be an arbitrary time instant. For $j \in\{0,1, \ldots, d\}$, define $x_{j}(k)$ as $\mathrm{T}(k-d+j)$, and for $j \in\{d+1, d+$ $2, \ldots, 2 d+1\}$, define $x_{j}(k)$ as $\mathrm{T}_{\mathrm{i}}(k-2 d-1+j)$. Also, let $x_{2 d+2}(k)$ be $\mathrm{T}_{\mathrm{c}}(k)$ and $y(k)$ be $\mathrm{T}_{\mathrm{o}}(k)$. Then, according to (5), (6) and (7), one can obtain the overall dynamics as

$$
\begin{aligned}
x_{0}^{+}= & x_{1}, \\
\vdots & \\
x_{d}^{+}= & \left(1-\frac{\theta_{2}}{\theta_{1}}\left(\mathrm{~F}_{\mathrm{d}}+\mathrm{F}_{\mathrm{r}}\right)-\theta_{3}\right) x_{d} \\
& \quad+\frac{\theta_{2}}{\theta_{1}}\left(\mathrm{~F}_{\mathrm{d}}+\mathrm{F}_{\mathrm{r}}\right) x_{2 d+1}+\theta_{3} x_{2 d+2}, \\
x_{d+1}^{+}= & x_{d+2}, \\
\vdots & \frac{\mathrm{F}_{\mathrm{d}}}{\mathrm{F}_{\mathrm{d}}+\mathrm{F}_{\mathrm{r}}} \mathrm{T}_{\mathrm{d}}+\frac{\mathrm{F}_{\mathrm{r}}}{\mathrm{F}_{\mathrm{d}}+\mathrm{F}_{\mathrm{r}}} \frac{1}{\theta_{1}}\left(x_{d+1}-x_{0}\right), \\
x_{2 d+1}^{+}= & \theta_{4}+\theta_{5} x_{2 d+2}+\theta_{6} x_{d}, \\
x_{2 d+2}^{+}= & \frac{1}{\theta_{1}}\left(x_{2 d+1}-x_{d}\right) .
\end{aligned}
$$

Let $\mathrm{x}$ denote the state vector of the system, $\mathrm{x}=$ $\left[x_{0}, x_{1}, \ldots, x_{2 d+1}, x_{2 d+2}\right]^{\top}$. One can write the dynamics of the system in (10) as

$$
\begin{aligned}
\mathrm{x}^{+} & =\mathrm{A}_{\theta}\left(\mathrm{F}_{\mathrm{d}}, \mathrm{F}_{\mathrm{r}}\right) \mathrm{x}, \\
y & =\mathrm{c}_{\theta}^{\mathrm{T}} \mathrm{x},
\end{aligned}
$$

where $c_{\theta}$ is a parametrized vector in $\mathbb{R}^{2 d+3}$ and $A_{\theta}\left(F_{d}, F_{r}\right)$ is a parameter dependent matrix-valued function with values in $\mathbb{R}^{(2 d+3) \times(2 d+3)}$ with arguments $\mathrm{F}_{\mathrm{r}}$ and $\mathrm{F}_{\mathrm{d}}$. If we substitute (9) in (11), we have the complete dynamics from input $u$ to output $y$.

\section{Identification of Plant and Parameter Estimation}

To identify the plant in (11) a prediction error minimization (PEM) method [7] is used to estimate the parameters $\theta_{1}, \cdots, \theta_{6}$ and $d$. The functions $f_{d}$ and $f_{r}$ in (9) are derived using a parametric non-linear regression approach.

Consider a general system with parametrized dynamics

$$
\begin{aligned}
\mathrm{x}^{+} & =\mathrm{F}_{\theta}(\mathrm{x}, \mathrm{v}), \\
\mathrm{y} & =\mathrm{G}_{\theta}(\mathrm{x}, \mathrm{v}),
\end{aligned}
$$

where $\theta, \mathrm{x}, \mathrm{v}$ and $\mathrm{y}$ are the vectors of parameters, states, control (and/or disturbance) inputs and outputs, respectively. The values of $\mathrm{x}(k), \mathrm{v}(k)$ and $\mathrm{y}(k)$ are measured and available for $k \in\left\{0,1,2, \ldots, N_{k}\right\}$. Also, let $K$ be a positive integer with $K \leq N_{k}$. For a fixed vector of parameters $\theta$ and given $\mathrm{x}(k)$ and $[\mathrm{v}(k+i)]_{i=0}^{K}=\left[\mathrm{v}(k)^{\top} \mathrm{v}(k+1)^{\top} \ldots \mathrm{v}(k+K)^{\top}\right]^{\top}$, one can predict state and output vectors for time instants $\{k+i \mid i=1, \ldots, K\}$, for any $k \leq N_{k}-K$. This can be done using the following iterative procedure

$$
\begin{array}{ll}
\hat{\mathrm{x}}(k \mid \theta, k) & =\mathrm{x}(k), \\
\hat{\mathrm{x}}(k+i+1 \mid \theta, k) & =\mathrm{F}_{\theta}(\hat{\mathrm{x}}(k+i \mid \theta, k), \mathrm{v}(k+i)), \\
\hat{\mathrm{y}}(k+i \mid \theta, k) & =\mathrm{G}_{\theta}(\hat{\mathrm{x}}(k+i \mid \theta, k), \mathrm{v}(k+i)),
\end{array}
$$

where $i=0,1, \ldots, K$. The resulting predicted value $\hat{\mathrm{y}}(k+$ $K \mid \theta, k)$ is called the $K$-step look-ahead output prediction. One can define the $K$-step look-ahead prediction cost function as

$J_{K}(\theta)=\frac{1}{N_{k}-K+1} \sum_{k=0}^{N_{k}-K}(\hat{\mathrm{y}}(k+K \mid \theta, k)-\mathrm{y}(k+K))^{2}$.

The $K$-step look-ahead prediction error minimization (PEM) problem is defined as $\min _{\theta \in \Theta} J_{K}(\theta)$, where $\Theta$ is the set of feasible parameters. The resulting parameter estimate is

$$
\theta^{*}=\operatorname{argmin}_{\theta \in \Theta} J_{K}(\theta) .
$$

Note that optimization problem (15) is in general non-convex which can be computationally demanding, particularly when the size of the parameter vector and the number of lookahead steps are large. In order to partially overcome these issues, as separate measurements of $\mathrm{T}_{\mathrm{i}}, \mathrm{T}_{\mathrm{c}}, \mathrm{T}_{\mathrm{o}}, \mathrm{T}_{\mathrm{d}}, \mathrm{F}_{\mathrm{i}}$ and $F_{d}$ are available, we can break down the PEM problem into subproblems with smaller parameter vectors. More precisely, using (5) and (2), or equivalently (10a) and (10d), we can establish a dynamical system similar to (12), where the state is $\mathrm{T}$, the vector of inputs is $\left[\mathrm{T}_{\mathrm{i}}, \mathrm{T}_{\mathrm{c}}, \mathrm{F}_{\mathrm{i}}\right]^{\mathrm{T}}$ and the output is 
$\mathrm{T}_{\mathrm{o}}$. Following that, we solve the $K$-step look-ahead PEM problem (15) to obtain $\theta_{1}^{*}, \theta_{2}^{*}$ and $\theta_{3}^{*}$. Similarly, based on (6) and (2), or equivalently (10c) and (10d), we can consider another dynamical system, where the vector of inputs is $\left[\mathrm{T}_{\mathrm{i}}, \mathrm{T}_{\mathrm{o}}\right]^{\top}$ and the state as well as output is $\mathrm{T}_{\mathrm{c}}$. Using $\theta_{1}^{*}$ and solving the $K$-step look-ahead PEM problem (15), one can obtain $\theta_{4}^{*}, \theta_{5}^{*}$ and $\theta_{6}^{*}$. Figure 2 compares the resulting estimates of these two identified dynamics for the $K$-step look-ahead prediction to experimental measurements for a validation operation cycle, using data not used for identification. Note that these PEM problems can be solved based only on the available historical measurement data and without knowledge of the delay $d$, which can be determined in a separate step.

The delay can be estimated based on (7) and (8) by finding the value for $d$, where we have the best match in the quantities. More precisely, one can estimate $d$ as the solution of the following problem

$$
\begin{aligned}
d^{*}=\operatorname{argmin}_{d \in \mathbb{N}} & \frac{1}{N_{k}-d} \sum_{k=d}^{N_{k}-1}\left(\mathrm{~T}_{\mathrm{i}}(k+1)\right. \\
& \left.-\frac{\mathrm{F}_{\mathrm{d}}(k) \mathrm{T}_{\mathrm{d}}(k)}{\mathrm{F}_{\mathrm{d}}(k)+\mathrm{F}_{\mathrm{r}}(k)}-\frac{\mathrm{F}_{\mathrm{r}}(k) \mathrm{T}_{\mathrm{o}}(k-d)}{\mathrm{F}_{\mathrm{d}}(k)+\mathrm{F}_{\mathrm{r}}(k)}\right)^{2} .
\end{aligned}
$$

Note that (16) is a one-step look-ahead PEM problem.

In order to find functions $f_{d}$ and $f_{r}$ in (9), we use a nonlinear regression. We model $f_{d}$ and $f_{r}$ as polynomials,

$$
\mathrm{f}_{\mathrm{d}}(u)=\sum_{i=0}^{n_{\mathrm{d}}} \theta_{i}^{(\mathrm{d})} u^{i}, \quad \mathrm{f}_{\mathrm{r}}(u)=\sum_{i=0}^{n_{\mathrm{r}}} \theta_{i}^{(\mathrm{r})} u^{i},
$$

where $\theta_{0}^{(\mathrm{d})}, \theta_{1}^{(\mathrm{d})}, \cdots, \theta_{n_{\mathrm{d}}}^{(\mathrm{d})}$ and $\theta_{0}^{(\mathrm{r})}, \theta_{1}^{(\mathrm{r})}, \cdots, \theta_{n_{\mathrm{r}}}^{(\mathrm{r})}$ are the corresponding coefficients for $f_{d}$ and $f_{r}$, respectively. Define vector $\theta^{(\mathrm{d})}$ as $\left[\theta_{i}^{(\mathrm{d})}\right]_{i=0}^{n_{\mathrm{d}}}$ and vector $\theta^{(\mathrm{r})}$ as $\left[\theta_{i}^{(\mathrm{r})}\right]_{i=0}^{n_{\mathrm{r}}}$, and also, for any given non-negative integer $n$, let $\phi_{n}: \mathbb{R} \rightarrow \mathbb{R}^{n+1}$ be a vectorvalued function defined as $\phi_{n}(u)=\left[1, u, u^{2}, \cdots, u^{n}\right]^{\top}$. Accordingly, we can write function $f_{d}$ and function $f_{r}$ as

$$
\mathrm{f}_{\mathrm{d}}(u)=\phi_{n_{\mathrm{d}}}(u)^{\top} \theta^{(\mathrm{d})}, \quad \mathrm{f}_{\mathrm{r}}(u)=\phi_{n_{\mathrm{r}}}(u)^{\top} \theta^{(\mathrm{r})} .
$$

The nonlinear regression problems can be written in form of ordinary least square problems. To find $f_{d}$, we solve the following optimization problem

$$
\theta^{(\mathrm{d})^{*}}=\operatorname{argmin}_{\theta^{(\mathrm{d})} \in \Theta^{(\mathrm{d})}}\left\|\mathbf{F}_{\mathrm{d}}-\Phi_{n_{\mathrm{d}}}(u)^{\top} \theta^{(\mathrm{d})}\right\|^{2},
$$
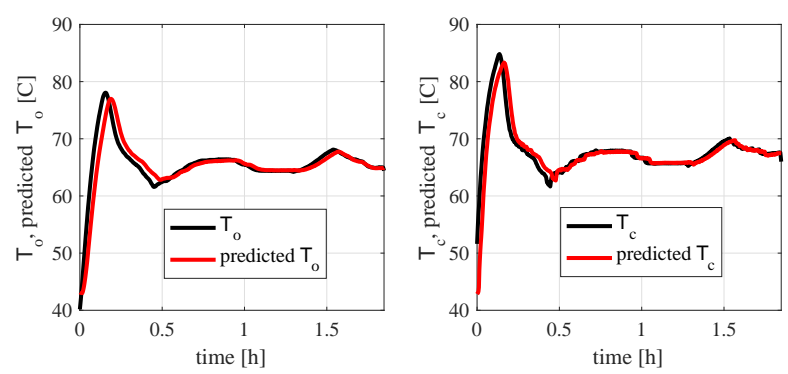

Fig. 2. Comparison of experimental measurements of $T_{o}$ and $T_{C}$ and their $K$-step ahead prediction with $K=12$ for a validation operation cycle.
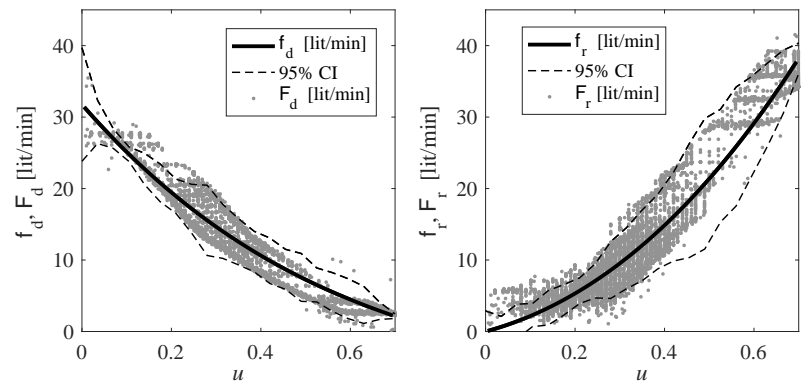

Fig. 3. Values of $F_{d}$ (left) and $F_{r}$ (right) versus the opening level of the mixing valve. The solid lines show the estimated functions $f_{d}$ (left) and $f_{r}$ (right), in (9), respectively. The gray dots correspond to measured data. The $95 \%$ confidence intervals are shown in dashed lines.

where $\Theta^{(d)}$ is the feasible set for candidate vectors of coefficients $\theta^{(\mathrm{d})}, \mathbf{F}_{\mathrm{d}}$ is vector $\left[\mathrm{F}_{\mathrm{d}}(0), \mathrm{F}_{\mathrm{d}}(1), \cdots, \mathrm{F}_{\mathrm{d}}\left(N_{k}\right)\right]^{\top}$, and $\Phi_{n_{\mathrm{d}}}(u)$ is matrix $\left[\phi_{n_{\mathrm{d}}}(u(0)), \phi_{n_{\mathrm{d}}}(u(1)), \cdots, \phi_{n_{\mathrm{d}}}\left(u\left(N_{k}\right)\right)\right]^{\top}$. Note that $\Phi_{n_{\mathrm{d}}}(u)$ is a Vandermonde matrix which is nonsingular when there are at least $n_{\mathrm{d}}+1$ different values amongst $u(0), \cdots, u\left(N_{k}\right)$. Analogously, the vector of coefficients corresponding to the polynomial representation of $f_{r}$ is derived based on a similar optimization problem. The resulting estimates of $f_{d}$ and $f_{r}$ are shown in Figure 3.

All of the identified plant parameters are given in Table I.

\section{PI-CONTROLleR TUNing PROBlem}

Consider the plant in simulation with the previously identified model in closed-loop form. Since the opening level of the mixing valve is limited, there is a saturation block at the loop defined as follows

$$
\operatorname{sat}(u)= \begin{cases}0, & \text { if } u \leq 0 \\ u, & \text { if } 0 \leq u \leq u_{\max } \\ u_{\max }, & \text { if } u_{\max } \leq u\end{cases}
$$

for a given maximum allowed valve opening ratio $0 \leq$ $u_{\max } \leq 1$. The controller is a PI-controller. Using a simple Euler's forward method, one can write the dynamics of the controller as

$$
\begin{aligned}
q^{+} & =q+T_{s} e, \\
u & =k_{p} e+k_{i} q,
\end{aligned}
$$

where $T_{s}$ is the sampling time, $k_{p}, k_{i}$ are the parameters corresponding to the controller, and $e$ is the error signal

\begin{tabular}{||c|l||c|l||}
\hline$T_{s}$ & $10[\mathrm{sec}]$ & $\theta_{6}^{*}$ & 1.6595 \\
$d$ & $4[\mathrm{sample}]$ & $\theta_{0}^{(\mathrm{d})^{*}}$ & $31.7616[\mathrm{lit} / \mathrm{min}]$ \\
$n_{\mathrm{d}}$ & 3 & $\theta_{1}^{(\mathrm{d}) *}$ & $-71.5796[\mathrm{lit} / \mathrm{min}]$ \\
$n_{\mathrm{r}}$ & 3 & $\theta_{2}^{(\mathrm{d}) *}$ & $53.5638[\mathrm{lit} / \mathrm{min}]$ \\
$\theta_{1}^{*}$ & 0.7005 & $\theta_{3}^{(\mathrm{d})^{*}}$ & $-16.8030[\mathrm{lit} / \mathrm{min}]$ \\
$\theta_{2}^{*}$ & $0.0015[\mathrm{~min} / \mathrm{lit}]$ & $\theta_{0}^{(\mathrm{r})}$ & $0[\mathrm{lit} / \mathrm{min}]$ \\
$\theta_{3}^{*}$ & 0.0826 & $\theta_{1}^{(\mathrm{r}) *}$ & $16.9685[\mathrm{lit} / \mathrm{min}]$ \\
$\theta_{4}^{*}$ & $24.97[\mathrm{C}]$ & $\theta_{2}^{(\mathrm{r})}$ & $45.9684[\mathrm{lit} / \mathrm{min}]$ \\
$\theta_{5}^{*}$ & -0.9133 & $\theta_{3}^{(\mathrm{r}) *}$ & $11.1483[\mathrm{lit} / \mathrm{min}]$ \\
\hline
\end{tabular}

Table I. Estimated values of plant parameters introduced in (10), (16) and (17). 
defined as $e=y-r$ where $r$ is the reference. Substituting parameters obtained in the previous section, in (9), (11) and (17), together with (20) and (21), one can write the dynamics for the total closed-loop system, which depends on the control parameters $\varphi:=\left[\begin{array}{ll}k_{i} & k_{p}\end{array}\right]^{\top}$, as

$$
\begin{aligned}
\mathrm{X}^{+} & =\mathrm{H}_{\varphi}(\mathrm{X}, \delta, r), \\
y & =\left[\mathrm{c}^{\top} 0\right] \mathrm{X},
\end{aligned}
$$

where $\mathrm{X}$ is the augmented state vector defined as $\left[\mathrm{x}^{\top} q\right]^{\top}$, $\delta$ is the signal of disturbances and $r$ is the fixed reference value, determined by the higher level controller. Accordingly, we denote the states and outputs of the system in one cycle of operation as

$$
\begin{aligned}
& \mathbf{X}(\varphi, \mathcal{D}):=[\mathrm{X}(t ; \varphi, \mathcal{D})]_{t=0}^{N} \in \mathbb{R}^{(2 d+3) \times N+1}, \\
& \mathrm{y}(\varphi, \mathcal{D}):=[y(t ; \varphi, \mathcal{D})]_{t=0}^{N} \in \mathbb{R}^{N+1},
\end{aligned}
$$

where $\mathcal{D}=\left([\delta(t)]_{t=0}^{N}, \mathrm{X}_{0}, r\right) \in \mathbb{R}^{n_{\mathcal{D}}}$ is a vector including the disturbances, initial states and the reference value. Here, the notation $[x]_{t=0}^{N}$ defines the concatenation of vectors $x(0)$, $x(1)$ up to $x(N)$ as $[x]_{t=0}^{N}=\left[x(0)^{\top} x(1)^{\top} \ldots x(N)^{\top}\right]^{\top}$.

Let $\Psi$ be a continuous real-valued function, where if $\mathbf{X}$ and $y$ are the states and outputs of the system in one cycle of operation, respectively, as defined in (23), then $\Psi(\mathrm{y}, \mathbf{X})$ is the corresponding performance of the system. For a choice of parameters $\varphi$, we can define the performance function $\Pi: \mathbb{R}^{n_{\varphi}} \times \mathbb{R}^{n_{\mathcal{D}}} \rightarrow \mathbb{R}$, a function of $\varphi$ and $\mathcal{D}$, as $\Pi(\varphi, \mathcal{D})=$ $\Psi(\mathrm{y}(\varphi, \mathcal{D}), \mathbf{X}(\varphi, \mathcal{D}))$ and the expected performance as

$$
\pi(\varphi)=\mathbb{E}_{\mathcal{D} \sim \mathbb{P}_{\mathcal{D}}}[\Psi(\mathrm{y}(\varphi, \mathcal{D}), \mathbf{X}(\varphi, \mathcal{D}))] .
$$

Here, $\mathbb{P}_{\mathcal{D}}$ describes the random nature of $\mathcal{D}$, i.e., $\mathcal{D} \sim \mathbb{P}_{\mathcal{D}}$, which is due to the disturbances in (9).

Similarly, the safe set can be defined using a continuous real-valued safety function, $\Xi$, i.e.,

$$
\Phi=\left\{\varphi \mid \xi(\varphi):=\mathbb{E}_{\mathcal{D} \sim \mathbb{P}_{\mathcal{D}}}[\Xi(\mathrm{y}(\varphi, \mathcal{D}), \mathbf{X}(\varphi, \mathcal{D}))] \geq 0\right\} .
$$

Then, the goal is finding $\varphi^{*}$, which maximizes the expected performance over controller parameters in the safe set, i.e., $\varphi^{*}=\operatorname{argmax}_{\varphi \in \Phi} \pi(\varphi)$. By restricting the controller parameters $\varphi$ to the safe set $\Phi$, i.e., $\varphi \in \Phi$, we can enforce desired properties of the closed-loop system such as safety and stability.

The performance function is given as black-box oracle rather than a closed-form formula, i.e., we can only obtain the value of performance, for a given $\varphi$, by means of performing an experiment or a simulation, as it is done in this paper. Moreover, the feasibility, i.e., if $\varphi \in \Phi$, can only be evaluated by performing experiments or simulations. This suggests obtaining the optimal parameter vector $\varphi^{*}$ using Bayesian optimization, which is introduced in the next section.

\section{Controller tuning Via BAyESian Optimization}

In this section, we present the implementation of safe active learning (SAL) [9] and Bayesian safe optimization (SafeOpt-MC) [2] for tuning the controller parameters in the closed-loop model (22).

Note that with the definitions of expected performance $\pi$ and safe set $\Psi$ in (24) and (25), respectively, we can consider

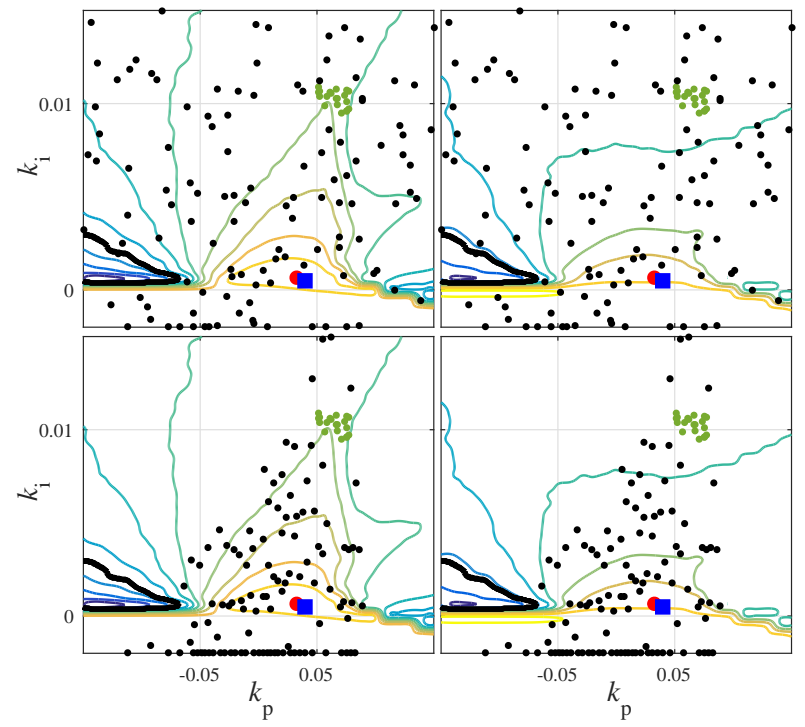

Fig. 4. The parameter region $\mathcal{R}_{\varphi}$ for SAL (top) and SafeOpt-MC (bottom) are shown. The contour plots correspond to the performance function, $\Pi$, (left) and the safety function, $\xi$, (right). The solid black curve shows the boundary of the safe-unsafe region. Initially, both of the algorithms start with same safe samples (green dots) and then iteratively sample from the parameter region (black dots). The final best samples (red solid dots), for both SAL and SafeOpt-MC, are close to the global best estimated parameters (blue squares).

almost arbitrary performance metrics and safety indicators. However, here we only consider a metric representing common issues of concerns such as overshoot and settling time. In this regard, we consider performance metric as

$\Psi(\mathrm{y})=-\alpha \max \left(\max _{0 \leq t \leq N}\left(y_{t}\right)-r, 0\right)-\frac{1-\alpha}{N+1} \sum_{t=0}^{N}\left|y_{t}-r\right|$,

where $\alpha$ is the trade-off between the minimizing the overshoot and the settling error. Large values at the output is not only unsafe but can also be an indication of instability. Therefore, we define the safety metric as the following constraint $\Xi(\mathrm{y})=\mathrm{y}_{\max }-\max _{0 \leq t \leq N} y_{t}$, where $\mathrm{y}_{\max }$ is the maximum tolerated output value. The objective is to maximize the performance metric without violating the safety constraint.

For the space of parameters, we consider a box region as $\mathcal{R}_{\varphi}:=\left[k_{p, \min }, k_{p, \max }\right] \times\left[k_{i, \min }, k_{i, \max }\right]$ in the $\left(k_{p}, k_{i}\right)$-plane. The performance as a function of the controller variables is optimized over this region such that the safety constraint is not violated. For this, we use SAL [9] and SafeOpt-MC [2]. For both of these algorithms we start with a safe starting point and some safe perturbations of it to define an initial safe set. Given this initial set, for each sample the output $y$ for one operation cycle is simulated using the previously identified model. With this the performance metric $\Psi(y)$ and safety metric $\Xi(y)$ are evaluated. Given all samples the Gaussian processes for the performance function and the safety set are derived as a function of the controller parameters $\varphi$. Figure 4 shows the $\left(k_{p}, k_{i}\right)$-points, which are sequentially evaluated with the algorithms in the search for the safe optimal point. It can be observed that both algorithms sample only from the safe set of parameters. For each new 


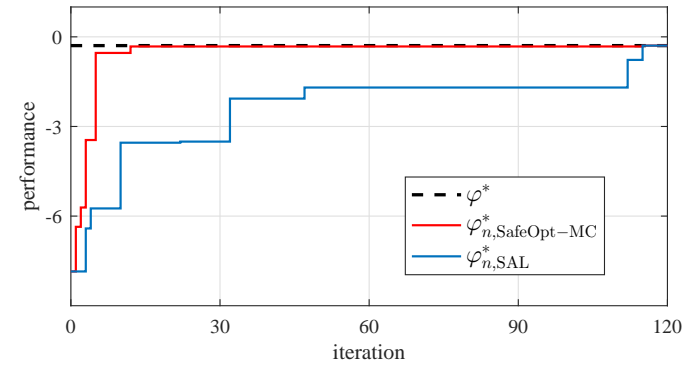

Fig. 5. The best observed sample up to iteration $n$ for both SAL and SafeOpt-MC converges to optimal value. The convergence for SAL is slower than for SafeOpt-MC

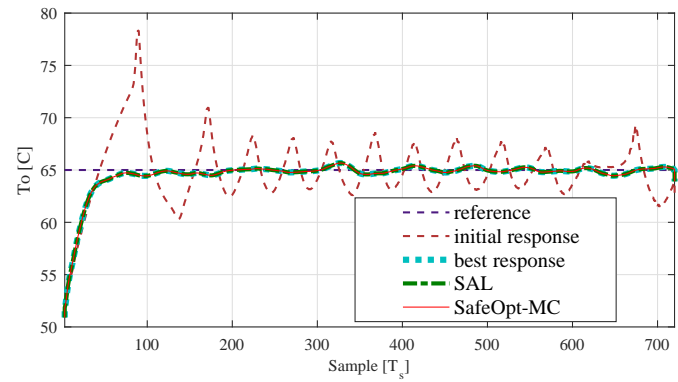

Fig. 6. The response of the system for the cases of not-tuned, tuned by SAL, tuned by SafeOpt-MC and the optimum parameters.

parameter sample, the Gaussian process is updated based on the resulting simulation output for this sample. While SAL mainly explores the parameter region, SafeOpt-MC trades-off between exploring $\mathcal{R}_{\varphi}$ and exploiting the collected samples to estimate the optimal parameters. As a result, SAL takes more scattered samples and reaches the optimal value after a higher number of sampling iterations. This can be seen from Figure 4 and Figure 5. In order to verify the result of these methods, we estimate the true optimal parameters. Therefore, we first grid $\mathcal{R}_{\varphi}$ finely and with respect to each point of the grid, we calculate the sample-based estimate of the expected performance and the expected safety function by performing 50 simulations. The contour plots in Figure 4 show the estimated values of performance function (left) and safety function (right). Figure 5 shows the performance of the best observed sample up to iteration $n$, which is denoted by $\varphi_{n, \mathrm{SAL}}^{*}$ for SAL and by $\varphi_{n, \mathrm{SafeOpt}-\mathrm{MC}}^{*}$ for SafeOpt-MC. One can see from Figures 4 and 5 that both of $\varphi_{n, \mathrm{SAL}}^{*}$ and $\varphi_{n, \mathrm{SafeOpt}-\mathrm{MC}}^{*}$ converge to the optimum safe parameters. We can also observe that the convergence for SAL is slower, as expected. Finally, the output of the system for the cases of not-tuned, i.e., using the initial safe parameters, tuned by SAL, tuned by SafeOpt-MC and the best possible output are shown in Figure 6. Since, the parameters resulting from SAL and SafeOpt-MC both converged to the optimal parameters, the corresponding responses coincide.

\section{CONCLUSION}

In this paper, the problem of controller tuning for an operating unit in a building energy system is considered. The problem is formulated in form of a black-box optimization. Following that, two candidate approaches, one based on safe active learning (SAL) and the other one based on safe Bayesian optimization (SafeOpt-MC) are considered for obtaining the solution. In order to assess the performances of these methods and the quality of their solutions, a physicsbased gray-box model of the plant is derived and the plant parameters are identified using available historical measurement data. The model obtained is used as an oracle in the controller tuning procedure in simulation. An intensive grid search verifies that the resulting iterative estimations of the controller parameters converge to the true optimal parameters, while respecting safety of the system during the controller tuning.

As a next step, the resulting controller parameters are to be tested on the real heat pump. Tuning the controller parameters in simulation here is reasoned by time issues. While in simulations thousands of cycles can be simulated in a second, the real heat pump considered is operated in average only for two cycles a day, which would result in an unacceptable tuning time for SAL of 60 days. With the comparison of the convergence time, these simulation results offer important insights for the experimental applicability of these methods for other heat pumps or similar systems in general, where SafeOpt-MC would be favored. Furthermore, with a simulation set-up as derived within this work, sample evaluations by experiment or simulation can be traded-off against each other in a joint tuning process for experimental verification on the one hand and time savings on the other hand.

\section{REFERENCES}

[1] B. W. Bequette, Process control: modeling, design, and simulation. Prentice Hall Professional, 2003.

[2] F. Berkenkamp, A. Krause, and A. P. Schoellig, "Bayesian optimization with safety constraints: safe and automatic parameter tuning in robotics," arXiv preprint arXiv:1602.04450, 2016.

[3] P. Cominos and N. Munro, "PID controllers: recent tuning methods and design to specification," IEE Proceedings-Control Theory and Applications, vol. 149, no. 1, pp. 46-53, 2002.

[4] A. Domahidi, F. Ullmann, M. Morari, and C. N. Jones, "Learning decision rules for energy efficient building control," Journal of Process Control, vol. 24, no. 6, pp. 763-772, 2014.

[5] C. Hang, K. Åström, and Q. Wang, "Relay feedback auto-tuning of process controllers-a tutorial review," Journal of process control, vol. 12, no. 1, pp. 143-162, 2002.

[6] J. Laustsen, "Energy efficiency requirements in building codes, energy efficiency policies for new buildings," International Energy Agency (IEA), vol. 2, no. 8, pp. 477-488, 2008.

[7] L. Ljung, System identification: theory for the user. Prentice-hall, 1987.

[8] M. Schillinger, B. Hartmann, P. Skalecki, M. Meister, D. NguyenTuong, and O. Nelles, "Safe active learning and safe Bayesian optimization for tuning a PI-controller," IFAC-PapersOnLine, vol. 50, no. 1, pp. 5967-5972, 2017.

[9] J. Schreiter, D. Nguyen-Tuong, M. Eberts, B. Bischoff, H. Markert, and M. Toussaint, "Safe exploration for active learning with Gaussian processes," in Joint European Conference on Machine Learning and Knowledge Discovery in Databases. Springer, 2015, pp. 133-149.

[10] B. Shahriari, K. Swersky, Z. Wang, R. P. Adams, and N. De Freitas, "Taking the human out of the loop: A review of Bayesian optimization," Proceedings of the IEEE, vol. 104, no. 1, pp. 148-175, 2016.

[11] D. Sturzenegger, D. Gyalistras, M. Morari, and R. S. Smith, "Model Predictive Climate Control of a Swiss Office Building: Implementation, Results, and Cost-Benefit Analysis," IEEE Trans. Control Syst. Technol., vol. 24, no. 1, pp. 1-12, 2016.

[12] Y. Sui, A. Gotovos, J. Burdick, and A. Krause, "Safe exploration for optimization with Gaussian processes," in International Conference on Machine Learning, 2015, pp. 997-1005. 